\title{
Novel Coronavirus (COVID-19)-Associated Guillain-Barré Syndrome: Case Report
}

\author{
Sandeep Rana, MD, FAAN, Artbur A. Lima, MD, Rabul Chandra, MD, \\ James Valeriano, MD, Troy Desai, MD, William Freiberg, DO, \\ and George Small, MD
}

Key Words: COVID-19, Guillain-Barré syndrome, dysautonomia, diplopia

( J Clin Neuromusc Dis 2020;21:240-242)

\section{INTRODUCTION}

The novel coronavirus infection (COVID-19) has spread rapidly throughout the world, causing considerable morbidity, mortality, ${ }^{1,2}$ and world health care system strain. Experience from China and Europe, the areas that were afflicted early in the pandemic, suggested that neurological complications consisted of headache, loss of smell, ${ }^{3}$ as well as taste, with some patients demonstrating altered mentation, dizziness, ataxia and rarely seizures, strokes, or skeletal muscle injury. ${ }^{4}$ There was an initial report from China describing a case of Guillain-Barré syndrome (GBS). ${ }^{5}$ We now present a similar case of GBS that developed soon after the patient contracted a COVID-19 infection.

\section{CASE}

From the Department of Neurology and Neuroscience Institute, Allegheny General Hospital, Allegheny Health Network, Pittsburgh, PA. The authors report no conflicts of interest.

Copyright $\odot 2020$ Wolters Kluwer Health, Inc. All rights reserved. our institution due to ascending limb weakness and numbness that followed symptoms of a respiratory infection. Two weeks before presentation, he initially developed rhinorrhea, odynophagia, fevers, chills, and night sweats. He stated that his wife had tested positive for COVID-19 and that his symptoms started soon after her illness. He was prescribed amoxicillin and a short course of steroids for 1 week by his PCP. At the end of this regimen, he developed watery diarrhea and sought medical attention at his local emergency department (ED). His stool tested positive for Clostridium difficile, and he was prescribed metronidazole. Over the next few days, he noted worsening paresthesias of his distal extremities bilaterally. His symptoms progressed to weakness of all limbs and difficulty voiding urine, which prompted him to return to the ED for further evaluation.

His medical history included hypertension, hyperlipidemia, restless leg syndrome, and chronic back pain. Medications included atorvastatin, diltiazem, pramipexole, lansoprazole, cyclobenzaprine, and naproxen. $\mathrm{He}$ was an ex-smoker, drank socially, and did not abuse recreational substances. He worked as a contractor.

On presentation to the ED, he developed progressive shortness of breath requiring intubation. Chest X-ray was negative other than an incidental finding of bibasilar lung infiltrates versus atelectasis. Testing for influenza A and B was negative. MRI of the thoracic and lumbar spine was reported to show no evidence of myelopathy or radiculopathy. He was transferred to our institution for suspicion of GBS.

On arrival to our hospital, his examina- tion demonstrated quadriparesis and areflexia with mute plantar responses. Further diagnostic evaluation included positive COVID-19 testing by RT-PCR. Lumbar puncture was deferred once the patient was confirmed to have COVID-19. He was started on a 5-day 
regimen of intravenous immunoglobulin $400 \mathrm{mg} / \mathrm{kg}$ daily for a presumed diagnosis of GBS. ${ }^{6}$ Oral vancomycin was started for $C$. difficile colitis. Hydroxychloroquine and azithromycin were also started for the COVID19 infection. His respiratory status improved, and he was extubated on hospital day 4. Because of his COVID-19 status, neurology follow-up was limited during the remainder of his admission, and electrodiagnostic studies were deferred. On hospital day 7 , he was discharged to an inpatient rehabilitation facility.

While in the inpatient rehabilitation, he was noted to have resting tachycardia and persistent difficulty urinating, which eventually required an indwelling catheter. $\mathrm{He}$ reported burning dysesthesias in his distal extremities and trunk, and complained of diplopia, which was worse on rightward gaze. Once his follow-up COVID-19 tests came back negative, neurology service performed detailed follow-up examination which was notable for facial diplegia, quadriparesis, and mild ophthalmoparesis, suggesting features of Miller Fisher syndrome. He was profoundly weak in his lower extremities with an MRC scale of $0-1 / 5$ in both proximal and distal muscles, and $3 / 5$ in proximal and $4 / 5$ in distal muscles of the upper extremities. His sense of smell and taste was intact. Testing for GQ1b was deferred, given that at his clinical stage, the results would not have changed management.

His EMG/nerve conduction studies performed 3 weeks after his onset of symptoms revealed abnormalities that met electrodiagnostic criteria for definite demyelinating polyneuropathy with multiple motor nerves showing prolonged distal latencies $(>150 \%$ of the upper limit of normal), conductions blocks, and slowing of conduction velocities ( $<70 \%$ of the lower limit of normal). $\mathrm{F}$ and $\mathrm{H}$ waves were absent as were all sensory responses, except the sural, which has been typically reported in patients with GBS. ${ }^{7}$ The needle portion of the study revealed evidence of abnormal insertional activity in the form of positive waves and fibrillation potentials being more prominent in the distal muscles of both upper and lower extremities, and normal-appearing motor unit potentials with decreased recruitment. Overall, electrical abnormalities were consistent with the demyelinating form of Guillain-Barré syndrome with secondary axonal degeneration.

\section{DISCUSSION}

We report a case of GBS that was preceded by COVID-19 infection. His deficits were characterized by quadriparesis, burning dysesthesias, mild ophthalmoparesis, and dysautonomia. Interestingly, he did not have the loss of smell and taste that has been documented in many COVID-19 patients. He briefly required mechanical ventilation and was successfully weaned after receiving a course of intravenous immunoglobulin.

The first case of COVID-19-associated GBS was reported from China. Subsequently, a case series of 5 patients was reported from Italy, ${ }^{8}$ suggesting that the incidence of Guillain-Barré syndrome is probably higher than initially appreciated. The unique clinical features of our case, namely urinary retention secondary to dysautonomia and ocular symptoms of diplopia, compared with cases reported in the literature highlight the variability in the clinical presentation of GBS associated with COVID19 infection.

Common to the cases reported in the literature as well as in our patient, the symptoms of GBS were noted to occur within days of the COVID-19 infection. This onset is similar to a case report of acute Zika virus infection with concurrent GBS, suggesting a parainfectious complication. ${ }^{9}$ Our case had typical electrophysiological findings of demyelinating polyneuropathy seen in patients with GBS, whereas the case series from Italy suggests that in COVID-19associated GBS, axonal variants could be just as common.
NEUROMUSCULAR

DISEASE

Volume 21, Number 4

June 2020 


\section{REFERENCES}

1. Wang D, Hu B, Hu C, et al. Clinical characteristics of 138 hospitalized patients with 2019 novel coronavirus-infected pneumonia in wuhan, China. $J A-$ MA. 2020. doi: 10.1001/jama.2020.1585.

2. Guan WJ, Ni ZY, Hu Y, et al. Clinical characteristics of coronavirus disease 2019 in China. $N$ Engl J Med. 2020;382:1708-1720.

3. Eliezer M, Hautefort C, Hamel A, et al. Sudden and complete olfactory loss function as a possible symptom of COVID-19. JAMA Otolaryngol Head Neck Surg. doi: 10.1001/jamaoto.2020.0832.

4. Mao L, Jin H, Wang M, et al. Neurological manifestations of hospitalized patients with coronavirus disease 2019 in wuhan, China. JAMA Neurol. 2020. doi: 10.1001/jamaneurol.2020.1127.
5. Zhao H, Shen D, Zhou H, et al. Guillain-Barré syndrome associated with SARS-CoV-2 infection: causality or coincidence? Lancet Neurol. doi: 10.1016/S1474-4422(20) 30109-5.

6. Fokke C, Van Den Berg B, Drenthen J, et al. Diagnosis of Guillain-Barré syndrome and validation of Brighton criteria. Brain. 2014;137:33-43.

7. Gordon PH, Wilbourne AJ. Early electrodiagnostic findings in guillain-Barré syndrome. Arch Neurol. 2001;58:913-917.

8. Toscano G, Palmerini F, Ravaglia, et al. Guillain Barré syndrome associated with SARS- CoV-2. NEJM 2020. doi: 10.1056/NEJMc2009191.

9. Siu R, Bukahari W, Todd A, et al. Acute Zika infection with concurrent onset of Gullain -Barré syndrome. Neurology. 2016;87:1623-1624. 